Invited Commentary for target article by Vikram K. Jaswal and Nameera Akhtar, "Being vs. Appearing Socially Uninterested: Challenging Assumptions about Social Motivation in Autism"

$\begin{array}{ll}\text { Word count, abstract: } & 60 \text { (guidelines } 60 \text { words max) } \\ \text { Word count, main text: } & 999 \text { (guidelines 1000 words max) } \\ \text { World count, references: } & 1221 \\ \text { Word count, entire text: } & 2221\end{array}$

Being Misunderstood in Autism: The role of motor disruption in expressive communication, implications for satisfying social relations

Jonathan Delafield-Butt ${ }^{1}$, Colwyn Trevarthen ${ }^{2}$, Philip Rowe ${ }^{1,3}$, Christopher Gillberg ${ }^{4}$

${ }^{1}$ Laboratory for Innovation in Autism, University of Strathclyde

2 School of Psychology, University of Edinburgh

3 Department of Biomedical Engineering, University of Strathclyde

${ }^{4}$ Gillberg Neuropsychiatry Centre, University of Gothenburg

\title{
Jonathan Delafield-Butt
}

Laboratory for Innovation in Autism, University of Strathclyde, 40 George Street, Glasgow G1 1QE, Scotland, UK

$+44(0) 1415483076$

jonathan.delafield-butt@strath.ac.uk

https://www.strath.ac.uk/staff/delafieldbuttjonathandr/

Colwyn Trevarthen

School of Psychology, University of Edinburgh, 3 Charles Street Lane, Edinburgh EH8 9AD, Scotland, UK

$+44(0) 1316513083$

c.trevarthen@ed.ac.uk

http://www.pmarc.ed.ac.uk/people/colwyntrevarthen.html

Philip Rowe

Department of Biomedical Engineering, University of Strathclyde, 40 George Street, Glasgow G1

$$
\text { 1QE, UK }
$$

+44(0) 1415483108

philip.rowe@strath.ac.uk

https://www.strath.ac.uk/staff/rowephilipprof/

Christopher Gillberg 


\title{
Gillberg Neuropsychiatry Centre, University of Gothenburg
}

Kungsgatan 12, SE- 411 19, Gothenburg, Sweden

$0046(0) 3134259700046$

christopher.gillberg@gnc.gu.se

https://gillbergcentre.gu.se/english/research-staff-\%26-associates/gillberg--christopher

\begin{abstract}
$\underline{\text { Abstract }}$
Jaswal and Akhtar's outstanding target article identifies the necessary social nature of the human mind, even in autism. We agree with the authors and present significant contributory origins of this autistic isolation in disruption of purposeful movement made social from infancy. Timing differences in expression can be misunderstood in embodied engagement, and social intention misread. Sensitive relations can repair this.
\end{abstract}

\section{$\underline{\text { Main Text }}$}

The human mind is not isolated, it is a dynamic relational subject animating the society in which it lives. Heidegger named this Mitsein, a state of 'being-with' that is the foundation of human experience. We are an ultra-social species, "obligatorily gregarious" (de Waal, 2006, p. 4).

Jaswal and Akhtar present an impressive array of evidence that individuals with autism retain a fundamental drive for social interaction and meaningful social relations. Mitsein in autism is intact, although its means of expression and social interaction are characteristically different. Recent research on the neuroscience of the human social brain, and affective 'moral' regulation of acts of social engagement, changes the theory of disorders of relating, including in schizophrenia and autism (Nieuwenhuys, 2012; Damasio, Damasio and Tranel, 2013)

In our work, we have examined the embodied nature of human social interaction in ontogenesis, measuring its origins in expressive intentions of the infant's subjective self (Delafield-Butt and Gangopadhyay, 2013; Delafield-Butt et al., 2018), made in relation to the movements of another (Delafield-Butt and Trevarthen, 2015; Trevarthen and Delafield-Butt, 2017a) that can be affected and contribute to pathology (Trevarthen et al, 2015). Feelings and desires expressed though a reciprocal coherence of felt action co-create social meaning (Trevarthen and Delafield-Butt, 2013a; Delafield-Butt and Trevarthen, 2015). After nine months an infant's acts of selective attention combine interests in objects and other persons (Hubley \& Trevarthen 1979; Aitken and Trevarthen 1997), which shapes learning the values of a culture (Trevarthen, 2009; Trevarthen \& DelafieldButt, 2017b).

In autism, we identify a fundamental disruption of the intuitive prospective motor control and its affective regulation in 'vitality dynamics' (Fournier et al, 2010; Stern, 2010). There is an alteration in the sub-second kinematic patterns of intentional movement of the arms and hands (Trevarthen \& Delafield-Butt, 2013b), whether to swing the arms, reach to touch, swipe a tablet or write a name (Cook at al, 2013; Torres, et al, 2013; Dowd et al, 2011; Anzulewicz et al, 2016; Grace et al., 2018).

Precise timing is required for efficient, purposeful movement and for effective expressive gesture in dialogue (Trevarthen, Delafield-Butt and Schögler, 2011). Child psychiatrist Daniel Stern called the affective nature of the action pattern 'vitality affects' (Stern, 2010), autonomic regulations expressed in movements shaped to convey visceral states of feeling (Damasio, 1999; Porges, 2011). 
The evidence shows these actions are timed differently in autism, and that perception of others' vitality affects is weakened (Rochat et al, 2013; Di Cesare et al, 2017). This perturbation of human communication and affectionate social engagement (Delafield-Butt and Trevarthen, 2013b) is expressed as autistic aloneness and self-protective isolation (St Claire, Danon-Boileau and Trevarthen, 2007), which can be misread as absence of sociability by persons with whom an autistic child is seeking meaningful engagement and shared learning (Trevarthen and Delafield-Butt, 2013b; de Jaegher, 2013; Cook, 2016).

A similar break in the reciprocal dynamic of the dyad is found in congenital Moebius syndrome that disrupts or paralyses facial expression, interrupting affective resonance. Moebius is associated with a high incidence of autism (Gillberg and Steffenburg, 1989). Other neurodevelopmental motor disorders, such as Deficits in Attention, Motor Control and Perception (DAMP) and Developmental Coordination Disorder (CDC), have autistic features. 'Motor clumsiness' and autism overlap (Gillberg, 1983; 2003; Gillberg and Kadesjö, 2003).

A likely site of the origins of this autism motor disturbance is in brainstem sensory and motor integrative systems that are closely coupled to those responsible for affective evaluation and social motor expression, together with closely coupled cerebellar structures (Trevarthen and DelafieldButt, 2013b; Coleman and Gillberg, 2012; Porges, 2011; Fatemi et al, 2012). Moebius syndrome follows a developmental error of the cranial nerves, and movement disturbance indicative of a brainstem growth error is evident in autism at birth (Teitelbaum et al., 1998), and in preschool children with autism (Bosco et al., 2018). Volumetric differences persist across the lifespan (Haar et al., 2014). Brainstem disruption affects one's core integration of psycho-motor experience, the so-called 'primary self' (Pansksepp \& Biven, 2012). It is not the wish for social engagement that is disrupted, but the coherence of primary sensory and motor information that make up the 'core self' expressed through body movement (Delafield-Butt and Trevarthen, 2017).

New theory of the 'social brain' gives importance to the forebrain systems centered on the insula, which develop as regulators of motor expressions of vital state and engagement of social affordances. Affective and social neuroscience is undergoing a change with recognition of the complexity of internal, basic proprioceptive and visceroreceptive evaluations of plans for action for the embodied self (Merker, 2007; Vandekerckhove and Panksepp, 2011), and for cooperative action in affectionate relations regulated by intersubjective sensitivity for these intrinsic parameters of consciousness with feeling (Shilbach et al, 2013). This approach supports transformations of psychological theory, with reduced dependence on linguistic communication and more appreciation of social meaning in embodied, non-verbal expression with 'vitality dynamics' to make meaningful contact. It supports an enhanced appreciation of shared awareness in 'the present moment' of what Damasio (2011) calls complex social emotional experiences, such as embarrassment, shame, guilt, contempt, compassion, and admiration. These will be complicated by abnormalities of motor coordination and timing that develop with autism.

Infants, like adults, avert eye-gaze in interactions that are felt to be too intense, a normal selfregulation (Jaffe et al., 2001). However, with autistics this behaviour may be appreciated differently. It is considered 'asocial' in literate, industrial cultures, which expect "conversational partners to respond promptly and to make their contributions to conversations unambiguous and relevant" (quoted by Jaswal and Akhtar from Grice, 1975). Conventions of motor expression in polite adult dialogues can affect the way autistic avoidance is received and cared for, and will influence the choice of therapy.

We are led by this thoughtful review to a re-evaluation of human understanding for a child's wellbeing, flourishing and developing. Meaningful social relations require sensitive appreciation and 
forms of response that respects all forms of expression, and seeks to share experiences (Sullivan and Rees, 2008). When this is achieved, understanding and satisfaction in relationship can flourish.

\section{$\underline{\text { Alphabetical reference list (APA standard) }}$}

Aitken, K. J., \& Trevarthen, C. (1997). Self-other organization in human psychological development. Development and Psychopathology, 9, 651-675.

Anzulewicz, A., Sobota, K., \& Delafield-Butt, J. T. (2016). Toward the autism motor signature: Gesture patterns during smart tablet gameplay identify children with autism. Scientific Reports, 6 . doi:10.1038/srep31107

Bosco, P., Giuliano, A., Delafield-Butt, J., Muratori, F., Calderoni, S., \& Retico, A. (2018). Brainstem enlargement in pre-school children with autism: Results from an inter-method agreement study of segmentation algorithms. Human Brain Mapping. in press

Coleman, M., \& Gillberg, C. (2012). The Autisms. Oxford: Oxford University Press.

Cook, J. L., Blakemore, S. J., \& Press, C. (2013). Atypical basic movement kinematics in autism spectrum conditions. Brain, 136(Pt 9), 2816-2824. doi:10.1093/brain/awt208

Cook, J. (2016). From movement kinematics to social cognition: The case of autism. Philosophical Transactions of the Royal Society of London B: Biological Sciences, 371(1693).

doi:10.1098/rstb.2015.0372

Damasio, A. R. (1999). The Feeling of What Happens: Body, Emotion and the Making of Consciousness. London: Heinemann.

Damasio, A. R. (2011). Neural basis of emotions. Scholarpedia, 6(3):1804. doi:10.4249/scholarpedia.1804

Damasio, A., Damasio, H., and Tranel, D. (2013). Persistence of feelings and sentience after bilateral damage of the insula. Cerebral Cortex, 23, 833-846. doi:10.1093/cercor/bhs077

De Jaegher, H. (2013). Embodiment and sense-making in autism. Frontiers in Integrative Neuroscience, 7. doi:10.3389/fnint.2013.00015

de Waal, Frans. (2006). Primates and Philosophers: How Morality Evolved. Princeton: Princeton University Press.

Delafield-Butt, J. T., \& Gangopadhyay, N. (2013). Sensorimotor intentionality: The origins of intentionality in prospective agent action. Developmental Review, 33(4), 399-425.

doi:10.1016/j.dr.2013.09.001

Delafield-Butt, J. T., \& Trevarthen, C. (2015). The ontogenesis of narrative: From moving to meaning. Frontiers in Psychology, 6. doi:10.3389/fpsyg.2015.01157

Delafield-Butt, J., \& Trevarthen, C. (2017). On the brainstem origin of autism: Disruption to movements of the primary self. In E. Torres \& C. Whyatt (Eds.), Autism: The movement sensing perspective: Taylor \& Francis CRC Press.

Delafield-Butt, J. T., Freer, Y., Perkins, J., Skulina, D., Schögler, B., \& Lee, D. N. (2018). Prospective control of neonatal arm movements: A motor foundation of embodied agency, disrupted in premature birth. Developmental Science, e12693.

Di Cesare, G., Sparaci, L., Pelosi, A., Mazzone, L., Giovagnoli, G., Menghini, D., . . Vicari, S. (2017). Differences in action style recognition in children with autism spectrum disorders. Frontiers in Psychology, 8(1456). doi:10.3389/fpsyg.2017.01456 
Dowd, A. M., McGinley, J. L., Taffe, J. R., \& Rinehart, N. J. (2012). Do planning and visual integration difficulties underpin motor dysfunction in autism? A kinematic study of young children with autism. Journal of Autism and Developmental Disorders, 42(8), 1539-1548.

doi:10.1007/s10803-011-1385-8

Fatemi, S. H., Aldinger, K. A., Ashwood, P., Bauman, M. L., Blaha, C. D., Blatt, G. J., .. . Welsh, J. P. (2012). Consensus paper: Pathological role of the cerebellum in autism. Cerebellum, 11(3), 777-807. doi:10.1007/s12311-012-0355-9

Fournier, K. A., Hass, C. J., Naik, S. K., Lodha, N., \& Cauraugh, J. H. (2010). Motor coordination in autism spectrum disorders: A synthesis and meta-analysis. Journal of Autism and Developmental Disorders, 40(10), 1227-1240. doi:10.1007/s10803-010-0981-3

Gillberg, C. (1983). Perceptual, motor and attentional deficits in swedish primary school children. Some child psychiatric aspects. Journal of Child Psychology and Psychiatry, 24(3), 377-403. doi:doi:10.1111/j.1469-7610.1983.tb00116.x

Gillberg, C. (2003). Deficits in attention, motor control, and perception: A brief review. Archives of Disease in Childhood, 88(10), 904-910. doi:10.1136/adc.88.10.904

Gillberg, C., \& Kadesjö, B. (2003). Why bother about clumsiness? The implications of having developmental coordination disorder (DCD). Neural Plasticity, 10(1-2), 59-68. doi:10.1155/NP.2003.59

Gillberg, C., \& Steffenburg, S. (1989). Autistic behaviour in moebius syndrome. Acta Paediatrica, 78(2), 314-316. doi:doi:10.1111/j.1651-2227.1989.tb11076.x

Grace, N., Johnson, B. P., Rinehart, N. J., \& Enticott, P. G. (2018). Are motor control and regulation problems part of the asd motor profile? A handwriting study. Developmental Neuropsychology, 1-14. doi:10.1080/87565641.2018.1504948

Haar, S., Berman, S., Behrmann, M., \& Dinstein, I. (2014). Anatomical abnormalities in autism? Cerebral Cortex. doi:10.1093/cercor/bhu242

Hubley, P., \& Trevarthen, C. (1979). Sharing a task in infancy. In I. Uzgiris (Ed.), Social Interaction During Infancy (pp. 57-80). San Francisco: Jossey-Bass.

Jaffe, J., Beebe, B., Feldstein, S., Crown, C., \& Jasnow, N. (2001). Rhythms of dialogue in infancy. Monographs of the Society of Research and Child Development., 66, 1-132.

Merker, B. (2007). Consciousness without a cerebral cortex: A challenge for neuroscience and medicine. Behavioral and Brain Sciences, 30, 63-134.

Nieuwenhuys R. (2012). The insular cortex: a review. Progress in Brain Research, 195, 123-63. PMID: 22230626 DOI.

Panksepp, J., \& Biven, L. (2012). The Archaeology of Mind: Neuroevolutionary origins of human emotions. New York: Norton.

Porges, S. W. (2011). The Polyvagal Theory: Neurophysiological Foundations of Emotions, Attachment, Communication, and Self-regulation. New York: WW Norton.

Rochat, M. J., Veroni, V., Bruschweiler-Stern, N., Pieraccini, C., Bonnet-Brilhault, F., Barthelemy, C., . . Rizzolatti, G. (2013). Impaired vitality form recognition in autism. Neuropsychologia,

51(10), 1918-1924. doi:10.1016/j.neuropsychologia.2013.06.002

Schilbach, L., Timmermans, B., Reddy, V., Costall, A., Bente, G., Schlicht, T., \& Vogeley, K. (2013). Toward a second-person neuroscience. Behavioral and Brain Sciences, 36(4), 393-414. doi:10.1017/s0140525x12000660

St Claire, C., Danon-Boileau, L., \& Trevarthen, C. (2007). Signs of autism in infancy: Sensitivity for rhythms of expression in communication. In S. Acquarone (Ed.), Signs of Autism in Infants: Recognition and early intervention. London: Karnac Books. 
Stern, D. N. (2010). Forms of Vitality: Exploring Dynamic Experience in Psychology, the Arts, Psychotherapy and Development. Oxford: Oxford University Press.

Sullivan, W. and Rees, J. (2008). Clean Language: Revealing Metaphors and Opening Minds. Carmarthen, Wales: Crown House Publishing, Ltd

Teitelbaum, P., Teitelbaum, O., Nye, O., Fryman, J., \& Maurer, R. G. (1998). Movement analysis in infancy may be useful for early diagnosis of autism. Proceedings of the National Academy of Sciences, U.S.A., 95, 13982-13987.

Torres, E. B., Brincker, M., Isenhower, R. W., Yanovich, P., Stigler, K. A., Nurnberger, J. I., .. . Jose, J. V. (2013). Autism: The micro-movement perspective. Frontiers in Integrative Neuroscience, 7. doi:10.3389/fnint.2013.00032

Trevarthen, C. (2009). The intersubjective psychobiology of human meaning: Learning of culture depends on interest for co-operative practical work and affection for the joyful art of good company. Psychoanalytic Dialogues, 19(5), 507-518. doi:10.1080/10481880903231894

Trevarthen, C., \& Delafield-Butt, J. T. (2013a). Biology of shared meaning and language development: Regulating the life of narratives. In M. Legerstee, D. Haley, \& M. Bornstein (Eds.), The Infant Mind: Origins of the social brain (pp. 167-199). New York: Guildford Press.

Trevarthen, C., \& Delafield-Butt, J. T. (2013b). Autism as a developmental disorder in intentional movement and affective engagement. Frontiers in Integrative Neuroscience, 7, 49. doi:10.3389/fnint.2013.00049

Trevarthen, C., \& Delafield-Butt, J. T. (2017a). Intersubjectivity in the imagination and feelings of the infant: Implications for education in the early years. In E. J. White \& C. Dalli (Eds.), Underthree year olds in policy and practice. New York: Springer.

Trevarthen, C., \& Delafield-Butt, J. T. (2017b). Development of consciousness. In B. Hopkins, E. Geangu, \& S. Linkenauger (Eds.), Cambridge Encyclopedia of Child Development (pp. 821-835). Cambridge: Cambridge University Press.

Trevarthen, C., Delafield-Butt, J. T., \& Schögler, B. (2011). Psychobiology of musical gesture: Innate rhythm, harmony and melody in movements of narration. In A. Gritten \& E. King (Eds.), Music and Gesture II (pp. 11-43). Aldershot: Ashgate.

Trevarthen, C., Aitken, K. J., Vandekerckhove, M., Delafield-Butt, J., \& Nagy, E. (2015).

Collaborative regulations of vitality in early childhood: Stress in intimate relationships and postnatal psychopathology Developmental Psychopathology (pp. 65-126): John Wiley \& Sons, Inc.

Vandekerckhove, M., \& Panksepp, J. (2011). A neurocognitive theory of higher mental emergence: From anoetic affective experiences to noetic knowledge and autonoetic awareness. Neuroscience and Biobehavioral Reviews, 35 (9), 2017-2025. 